\title{
Digital skills and teaching performance
}

Competencias digitales y desempeño docente

\section{Gladys Erika Lopez Cordova}

Magíster en Administración de la Educación, Universidad César Vallejo, https://orcid.org/0000-0003-1171-8723, gelopezc@ucvvirtual.edu.pe

Revista Iberoamericana de la Educación

Vol-Especial 12021

e-ISSN: 2737-632x

Abstract: The progress of technology and the arrival of the pandemic in the world has generated a large-scale replication in digital education, transforming education from the face-to-face environment to the virtual environment. The objective of this article is to review the progress regarding key points of digital competence and teaching performance. It is a theoreticaldescriptive exploration of a documentary type, with documents between 2019 and 2020. SciELO, Scopus and Web of Science databases were used, including descriptors such as "Digital competences", "pedagogical performance", "Digital inclusion" and " uses of TIC". These descriptors were combined in different ways at the instant of navigation in order to broaden the search criteria. In today's context, all educational institutions have had to migrate to the digital age, both public and private, as key to surviving in times of crisis, having technology as allies. Concluding, that teachers have had to adapt to this new educational and training profile in which we find ourselves engulfed by the technological trend in education within this teaching and learning scenario in an increasingly digital world.

Key words: Digital skills, pedagogical performance, digital inclusion, uses of TIC 
Resumen: El progreso de la tecnología y la llegada de la pandemia en el mundo ha generado una gran réplica a gran escala en la educación digital, transformando la educación del entorno presencial al entorno virtual. El presente artículo tiene por objetivo hacer una revisión sobre los avances referidos a puntos clave de competencia digitales y desempeño docente. Es una exploración teórico-descriptiva de tipo documental, con documentos comprendidos entre 2019 y 2020. Se utilizaron bases de datos SciELO, Scopus y Web of Science, incluyendo descriptores como "Competencias digitales", "desempeño pedagógico", "Inclusión digital” y "usos de las TIC". Estos descriptores fueron combinados de distintas formas al instante de la navegación con el propósito de agrandar los criterios de búsqueda. En el contexto de hoy que vivimos, todas las instituciones educativas han tenido que migrar a la era digital tanto públicas y privadas como clave para sobrevivir en tiempos de crisis teniendo como aliados a la tecnología. Concluyendo, que los docentes han tenido que adaptarse a este nuevo perfil educativo y formativo en el que nos encontramos sumido por la tendencia tecnológica en la educación dentro de este escenario de enseñanza y aprendizaje en un mundo cada vez más digital.

Palabras clave: Competencias digitales, desempeño pedagógico, Inclusión digital, usos de las TIC

\section{INTRODUCTION}

En el contexto actual frente a la crisis ocasionado por la pandemia, el mundo cambió y junto con ello la educación, que hoy representa grandes retos para 
los países en desarrollo como el nuestro que tienen la necesidad de reformular la educación para el modelo actual de la era digital.

Existen problemas relacionados con la dificultad de brindar servicios educativos de manera remota. Según las observaciones, las instituciones educativas públicas no tienen plataformas digitales para atender las necesidades de los alumnos, además, los docentes aún no están preparados para ello. Con la nueva realidad tecnológica y la falta de equipamiento técnico para realizar las tareas docentes, muchas personas tienen que adaptarse a su propia realidad.

La enseñanza digital se ha incrementado donde el papel que juega el docente en los entornos de enseñanza virtual es esencial. El adaptarse abruptamente a esta cultura digital los docentes han tenido que capacitarse e innovarse para los nuevos modelos de enseñanza y exigencias que requiere los perfiles frente a la revolución tecnológica actual. Como bien lo menciona Pol (2015, p.20) "Dentro del modelo de educación virtual se definen permisos de docentes, como facilitadores, e-tutores, que conocen la parte técnica, y a la vez tienen la capacidad de orientar a los alumnos". Se refiere a que los docentes asumen retos y los orientan según su dirección y acompañamiento, mientras que los estudiantes tienen un papel protagonista en la era digital.

La educación ha dado un giro inesperado cambiando todo nuestro panorama en la forma de educar y adquirir conocimiento tornándose todo en un entorno digital. Las TIC han aportado nuevo alfabetismo que potencian capacidades y competencias propias del siglo XXI. Estas culturas se ejercitan principalmente en la práctica digitales de los jóvenes en entornos de aprendizaje informal, principalmente en lugares y tiempo de ocio. (Busque, Medina y Ballano, 2013).

La educación tiene grandes desafíos por cumplir en tiempos de pandemia, con respecto a la inclusión digital, que involucra a la sociedad educativa en 
conjunto y debe formar parte de las aulas y en su formación del docente (Gil \& Cano, 2020). Por otro lado, las TIC han tenido una gran repercusión en la educación y su uso ha ido extendiendo a medida que la sociedad y la tecnología ha evolucionado hasta convertirse en una condición necesaria para que profesores y alumnos impartan e intercambien conocimientos (Hernández, Orrego \& Quiñones, 2018).

\section{MATERIALS AND METHODS}


El presente trabajo es una exploración teórico-descriptiva de tipo documental, ya que el trámite supone el rastreo, organización, sistematización y examen de un grupo de documentos electrónicos sobre el tema competencia digitales y desempeño docente en el lapso comprendido entre 2019 y 2020.

Las entidades de examen fueron todos esos documentos sobre el tema, encontrados en las bases de datos SciELO, Scopus y Web of Science. Como criterios de búsqueda, se incluyeron los próximos descriptores: “Competencias digitales", "desempeño pedagógico", "Inclusión digital” y "usos de las TIC". Estos descriptores fueron combinados de distintas formas al instante de la navegación con el propósito de agrandar los criterios de búsqueda.

Al hacer la búsqueda de los documentos, en todas las bases de datos, se preseleccionaron 25 artículos, de los cuales se escogieron siete, según con los criterios de incorporación y exclusión. No se han tomado en consideración para el examen esos artículos que no hacían referencia a los núcleos temáticos y/o esos que no estaban en revistas indexadas.

Para la organización de los documentos, se desarrolló en Excel una base de datos, con los próximos campos (categorías de análisis): encabezado del artículo, creador, año, revista, información de la revista, inconveniente de exploración, objetivos, tipo de exploración, procedimiento, especificación y tamaño de la exhibe, instrumentos usados, resultados y núcleo temático. 
Una vez estructurada la información, se agruparon los documentos en 4 núcleos temáticos a saber: competencias digitales, desarrollo pedagógico, Incorporación digital, usos de las TIC. Más adelante, se llevó a cabo el examen de todos los núcleos temáticos, identificando los inconvenientes abordados, formas, instrumentos, población y resultados, definiendo los más importante y describiendo los puntos recurrentes y divergentes entre los documentos seleccionados, por medio de un ejercicio de comparación recurrente. Por último, se llevó a cabo un examen global, se identificaron las convergencias y divergencias de todos los núcleos temáticos y se formularon conclusiones.

\section{DISCUSSION}

\section{Competencia desde el contexto digital:}

En pleno siglo XXI, se da un gran salto de lo presencial a la era digital, obligados bajo el contexto de crisis sanitaria que hoy vivimos, este cambio hace que la sociedad sea más competitiva y tenga a la educación abierto de par en par desde cualquier lugar y en tiempo real teniendo como aliado al internet. También mencionaremos que en esta vertiente digital nacen dos términos uno de competencia digital del estudiante y competencia digital docente (Gisbert, González \& Esteve, 2016).

En cuanto a, competencias digitales y educación según lo mencionado por Lévano et. al (2019), el incremento basado en las nuevas tecnologías y sus diferentes usos y aplicaciones han dado un giro en la sociedad y en el ambiente de la educación paras satisfacer las exigencias y cubrir la demanda que exige la educación actual. Bajo este paradigma señalamos el compromiso de los educadores debe estar a la par con los avances tecnológicos para satisfacer la necesidad de un mundo globalmente sumergido en la era digital.

\section{Competencias digitales}


La Competencia Digital (CD) supone la utilización creativo, crítico y seguro de las tecnologías de la información y la comunicación para conseguir trabajo, empleabilidad, estudio, uso del momento de libertad, tolerancia y participación en la sociedad.

Competencias digitales según Marza y Cruz (2018) son herramientas muy útiles que pueden ajustar dinámicas, conocimientos y procesos; a través de este curso, los alumnos consiguen capacidades para fomentar la transferencia de conocimiento y la originalidad. En este punto de vista, para Iordache, Mariën y Baelden (2017) propusieron asumir la competencia digital como el resultado más práctico y medible del proceso de formación relacionado con la nueva alfabetización digital.

En tanto; Ocaña, Valenzuela y Garro (2018) al resumir correctamente lo que creen que son las habilidades digitales, los antecedentes actuales deben ser concebidos en la aplicación de las TIC y la plataforma Interacción en tiempo real.

\section{Papel del docente en la era digital}

En relación con el papel del docente en la era digital es esencial, porque será el guía de encaminar a los estudiantes por el sendero del mundo de adquirir conocimiento y enriquecerse del mismo. Por lo que el docente marca su propia historia frente a estos hechos acontecidos por la pandemia y se reinventa y adapta rápidamente para enfrentarse a este mundo competitivo digital. Bajo este contexto el nuevo entorno de trabajo ha generado dificultades, según su edad muchos docentes presentaban dificultades para adaptarse a esta forma de enseñanza en la que los educadores no solo deben de estar alfabetizados digital si no contar con las herramientas adecuadas para potenciar su uso de la TIC y tener un buen desempeño profesional (Esteve, Gisbert \& Lázaro, 2016). 
El año 2020 sin duda, será recordado como el gran salto de la humanidad que pasó de la educación tradicional más conocida como educación presencial o convencional al lado opuesto que es la educación virtual o enseñanza en línea donde el alumno es el principal protagonista en adquirir conocimiento. El docente es piloto que conduce hacia el sendero de enseñar y aprender. En tanto, Bates (2015), en su libro titulado Teaching in a Digital Age, relata cómo el docente debe hacer uso a los medios tecnológicos que están a la mano para modernizar su metodología para impartir enseñanza y el aprendizaje del alumnado. Por lo tanto, la tecnología es el centro donde gira el aplicar e innovar las metodologías de enseñanza, y el docente se renueva en su papel frente al aula.

\section{Competencia digital pilar fundamental del siglo XXI}

En los últimos años la educación digital ha abarcado una gran notoriedad y protagonismo influyendo de modo directo en el aprendizaje tanto para docente como estudiante. Estamos viviendo en una era tecnológica donde la población a muy temprana edad ya dispone de un dispositivo móvil, computadora, tablet que generan voluntaria e involuntaria su red digital. Varios autores mencionan bajo este contexto, que el haber nacido con la tecnología y tener contacto directo desde muy temprana edad con este, no es garantía de tener habilidades tecnológicas homogéneas dentro de este periodo etario (Bossolasco, Chiecher y Dos Santos, 2020; Chiecher, 2019; Chiecher y Melgar, 2018; Chiecher, 2018; Gallardo et al., 2016; Kennedy, Judd, DalgarnoyWaycott, 2010; entre otros). Sin embargo, Chiecher y Riccetti (2019) en su experiencia realizada que pone en uso de WhatsApp y Google Docs donde los estudiantes tenían que realizar su tarea académica, observaron que mostraron escaso desempeño para comunicarse virtualmente y así como redactar colaborativamente en un archivo compartido. 
En el mundo actual donde la sociedad está conectada y constantemente adquiere aprendizaje y retroalimenta de conocimiento es requisito primordial que el educador adquiera nuevas capacidades técnicas y cognitivas para llevar a cabo a los enormes desafíos del conocimiento tecnológico, por lo cual, la rivalidad digital consigue un papel principal en todos los puntos del estudio durante la vida (Gisbertet al., 2016).

\section{Desempeño docente}

Frente los veloces cambios de la sociedad de hoy, es requisito ofrecer prioridad a la perspectiva de la política didáctica formulada por el Ministerio de Educación; pero principalmente quienes creen que se requieren cambios profundos en la destreza docente. El desarrollo docente y su reevaluación son trascendentales y esenciales para la creación de algún cambio de política didáctica. La optimización de la calidad didáctica debe tener en cuenta, primordialmente, el cambio de pensamiento del papel del maestro en la escuela.

Por lo tanto, además de establecer estándares claros en el proceso de preparación de los estudiantes para el aprendizaje, la reformulación de reglas públicas en el tema educativo para hacer mejor el estudio de los alumnos, además se considera una guía didáctica importante. El desempeño docente es el nuevo desafío del siglo XXI, la pandemia de coronavirus obligó a los centros educativos a cerrar y se suspendió las clases presenciales dando paso a la enseñanza remota de emergencia. La vida diaria de millones de jóvenes y adolescentes en edad escolar a nivel mundial se ve interrumpida o perturbada, y las situaciones nos han obligado a accionar con eficacia para llevar a cabo frente a un derecho básico que no puede detener llamada educación. 
En este caso, las demandas laborales de los docentes han aumentado y, al mismo tiempo, se les pide de manera rápida que adapten sus cursos presenciales a la interacción virtual o remota.

Este es un momento histórico que requiere un análisis detenido del significado de la enseñanza durante la pandemia, en este caso aún no estamos preparados, lo que repercute en el desempeño del docente.

Requerimos impulsar las capacidades de los docentes y alumnos de pedagogía para que logren tomar elecciones sobre la lección y el currículo en funcionalidad de sus alumnos, antecedentes, elementos, etc. Esto quiere decir ser responsable del trato desigual de los alumnos y tener en cuenta resoluciones creativas y ejecutables para cada circunstancia. Las utilidades virtuales no son para todos, debemos buscar novedosas elecciones. Esto necesita una más grande elasticidad y soberanía en la red social escolar.

\section{Evaluación del desempeño docente}

El desempeño docente es una práctica observable, que actúa en la aptitud del docente para expresarse y se relaciona con el logro de estudio esperado; o sea, la intención de la educación y la ejecución de las tareas asignadas dependen de la calidad y formación inicial del docente.

Los diferentes factores están encaminados a lograr un excelente nivel educativo (Benítez, Kabai y Encarada, 2017).

El tema de la evaluación del desarrollo docente es complicado y su aplicación varía según las zonas de todo el mundo y los sistemas políticos que ya están. (Rivas, 2015; Vaillant, 2016). Según Armando, González, y Paredes (2020) en el avance de la vivencia de estudio a distancia, el papel de los profesores es crucial en una crisis de salud por lo cual se debe determinar el nivel específico de conocimientos previos sobre la aplicación de las NTIC al desarrollo de la clase no presencial, es de vital importancia promover el trabajo colaborativo de formación docente y orientación profesional, y 
facilitar el acceso un equipo digital estable que le permite superar las barreras técnicas y de conexión para el buen desempeño docente.

Gálvez, E. \& Milla, R. (2018) menciona que el modelo de evaluación docente contribuye en un mejor desempaño docente relacionados con el aprendizaje que repercute en el marco del buen desempeño. En otras expresiones, en esas academias, los docentes que están metidos en la instrucción de la evaluación se desempeñarán mejor (Olaguibel, 2010).

Para Cárdenas, Soto-Bustamante, Dobbs-Díaz y Bobadilla (2012) concluyeron que los docentes tienen que enseñar y llevar a cabo, bien supuestamente pedagógica, pero más en el campo de los contenidos relacionados.

En el sistema de evaluación integrado a la política pública, se reconoce que los docentes son el motor de cambio y pueden proponer estrategias o modelos para resolver múltiples problemas. (Manzi, González y Sun, 2012).

Gracias a las ideas de organismos de todo el mundo, se ha incrementado el avance de reglas educativas en Latinoamérica y el Caribe, como la UNESCO, el Banco Mundial y el Banco Interamericano de Desarrollo. No obstante, en los años anteriores, el sistema educativo solamente ha sistematizado el desarrollo de evaluación del desarrollo docente (Melgar, 2015).

En Perú, se ha establecido una herramienta de trabajo para estructurar el buen hacer de los docentes de escuela, denominada marco de buen desempeño docente, que considera cuatro aspectos, a saber, nueve competencias y cuarenta desempeños. Estos aspectos son logros importantes para el país en la mejora de las prácticas docentes, a su vez, son elegibles para ser considerados asuntos públicos de vital importancia para el bienestar público. Población (Ministerio de Educación, 2012).

El marco legal para la evaluación del desempeño en el país es reciente. Para evaluar la efectividad de la enseñanza se deben establecer los estándares y 
métodos establecidos en las instituciones legales. En esta investigación se hace un breve análisis de la Ley General de Educación, Ley $\mathrm{N}^{\circ}$ 28044, la Ley de Reforma Docente, Ley N ${ }^{\circ} 29944$ y el Plan Nacional de Educación 2021; objetivo estratégico 3. Bien capacitados Profesores profesionales. En definitiva, para establecer un modelo, para realizar una evaluación, se debe estimar un marco legal relevante, que debe atender las necesidades educativas del país y considerar la reevaluación de la profesión docente. Actualmente, el Ministerio de Educación ha realizado esfuerzos para el proceso de planificación para mejorar el proceso de educación en tiempos de pandemia; a través de la formación docente, profesionalización y capacitaciones.

Esto significa que el país a través del gobierno central, los gobiernos regionales y Local, debe asegurarse de que haya una forma de aprendizaje a distancia durante este período Inmovilización social obligatoria, todos acceder a la educación, Indica que la enseñanza se ajusta al contenido de enseñanza de alta calidad del sistema.

\section{Acerca De La Inclusión Digital}

La inclusión digital en la educación es una herramienta potente para estar conectados mediante la red, en estos tiempos de pandemia son un derecho fundamental y representan un gran desafío para el estado, es una brecha que se tiene que ir acortando para brindar un mejor servicio y llegar a todos los rincones y en especial hacia los no conectado.

En tiempos de pandemia la educación hoy en día es un lujo, podemos apreciar la de enorme desigualdad que se ve plasmado especialmente en las zonas rurales que carecen de los requisitos esenciales para estar conectados, debido a necesidades en implementación de equipos informáticos o dispositivos móviles se presentan dificultades tanto para el docente como el alumno para acceder y poder desarrollar las clases por medio de las aulas virtuales en tiempos de pandemia. Con la nueva modalidad de desarrollar las clases 
utilizando el entorno virtual y con experiencia previamente adquiridas los docentes y el estado se alistan para que los ciudadanos se beneficien del entorno digital y contribuir una mejor educación ciudadana. Según McKnight (2016), los profesores deben utilizar la tecnología en el aula, esto puede ayudarlos a mejorar sus conocimientos y habilidades. Basilotta, Martín y García (2017) mencionaron la aplicación algunos de los proyectos que utilizan las TIC han impulsado los estudiantes estimulan su interés por aprender y desarrollan diversos Habilidades del curso. Álvarez y Cervera (2015) informaron que los docentes de este nivel secundario deben tener la capacidad, el talento y las habilidades para saber cómo usar la tecnología y comunicarse en las aulas aula.

\section{Acerca del Usos de las TIC.}

La tecnología de la información y comunicación, son los mejores aliados para el docente en tiempos de pandemia, mediante su kit de herramientas de TIC, han podido brindar educación de calidad. Basilotta, Martín y García (2017) mencionaron la aplicación de algunos de los proyectos que utilizan las TIC han impulsado a los estudiantes a estimular su interés por aprender y desarrollan diversas habilidades del curso. Las Tecnologías de la información y las contribuyen en el docente dentro de la didáctica y aprendizaje. El uso de las tecnologías de la información y la comunicación en diversos campos ha dado lugar a debates sobre los pros y los contras que podría significar su inclusión en la educación. Hoy en día si buscamos que el país avance necesitamos que su población sea alfabetizada digitalmente. Dentro del enfoque educativo aún existen lo no conectados, llamamos aquellas sociedades en la cual aún tiene la barrera de no formar parte de la población que tiene el servicio de internet para realizar sus actividades ya sea de forma, profesional, social, estudiantil. En muchos lugares recónditos del Perú profundo, se ha observado que profesionales en el ámbito de educación como 
son los maestros no cuentan con la implementación de instrumentos como, por ejemplo, una laptops, PC o celular para acceder a realizar sus clases y poder llegar a sus estudiantes mediante el uso de las TIC y brindar educación de calidad. 


\section{CONCLUSIONS}

Las competencias digitales son de vital importancia para el buen desempeño de los profesionales de la educación. Según Unesco (2016) el aporte de las TIC contribuye al acceso universal en educación, garantizando integración y calidad.

Necesitamos profesionales capacitados para en el manejo del uso de plataformas digitales para poder llegar a los estudiantes que se encuentran conectados desde los diferentes puntos del país en especial en las zonas rurales.

El gran reto del estado es llegar a más estudiantes, cubrir las necesidades de la población vulnerable que hoy en día forma parte de los no conectados y representa un gran desafío en tiempos de pandemia, teniendo como resultado muchos alumnos que han desertado el año académico.

Las TIC, juegan un rol muy importante dentro de la sociedad, son un gran aliado para los profesionales de la educación, mejoran sus competencias digitales y por ende el desempeño.

Por lo anterior, se menciona en brindar todos los recursos necesitados tanto en el aspecto didáctico y tecnológico para lograr los objetivos propuesto en cuanto a brindar una educación de calidad e igualdad de oportunidades en educación para todos. 


\section{REFERENCES}

Álvarez, J.y Cervera.M, (2015). Information Literacy Grade of Secondary School Teachers in Spain - Beliefs and Self-Perceptions. Revista Comunicar 45: La comunicación en un mundo que envejece (Vol. 23 - 2015). https://doi.org/10.3916/C45-2015-20

Armando, G; González, G; Paredes J. (2020) Desempeño y formación docente en competencias digitales en clases no presenciales durante la pandemia COVID-19. Doi: https://orcid.org/0000-0002-1668-5951

Basilotta,V.; Martín,M. y García,A.(2017) Project-based learning (PBL) through the incorporation of digital technologies: An evaluation based on the experience of serving teachers. Revista Computer and Human Behavior, Vol. 68, págs.501-

512.https://www.sciencedirect.com/science/article/pii/S074756321630805

6

Bates, T. (2015). Teaching in a Digital Age. Disponible: http://goo.gl/NAvukU

Benítez, J. E. M., Cabay, L. C. C., \& Encalada, V. D. G. (2017). Formación inicial del docente de educación física y su desempeño profesional. EmásF: Revista digital de educación física, 8(48), 83-95. https://emasf.webcindario.com/Formacion_inicial_del_docente_de_ EF_y_su_desempen o_profesional.pdf

Bossolasco, M. L., ChiecherA. C. y Dos Santos, D. (2020). Perfiles de acceso y apropiación de TIC en ingresantes universitarios. Estudio comparativo en dos universidades públicas argentinas. Pixel-Bit Revista de Medios y Educación, 57 , 151-172. https://recyt.fecyt.es/index.php/pixel/article/view/72199/48044

Busquet-Duran, J., Medina-Cambrón, A. y Ballano-Macías, S. (2013). El uso de las TRIC y el choque cultural en la escuela. Encuentros y desencuentros entre 
maestros y alumnos. Revista Mediterránea de Comunicación, 4 (2), 115 135. Doi: 10.14198/MEDCOM2013.4.2.06.

Cárdenas Pérez, A. V., Soto-Bustamante, A. M., Dobbs-Díaz, E., Bobadilla Goldschmidt, M. (2012). El saber pedagógico: componentes para una reconceptualización. Educ. Educ. Vol. 15, No. 3, 479-496. https://www.redalyc.org/pdf/834/83428627008.pdf

Chiecher, A. (2019). Culturas juveniles y prácticas docentes ¿Sintonía o desajuste?. Conferencia presentada en el VIII Seminario Internacional de Educación a Distancia. Universidad Nacional de Jujuy, Tilcara. https://cerac.unlpam.edu.ar/index.php/praxis/article/view/4259

Chiecher, A. (2019). Competencias digitales en estudiantes de nivel medio y universitario. ¿Homogéneas o heterogéneas? Praxis educativa. DOI: https://doi.org/10.19137/praxiseducativa-2020-240208

Chiecher, A. y Melgar, M. F. (2018). ¿Lo saben todo? Innovaciones educativas orientadas a promover competencias digitales en universitarios. Revista Innovación Educativa, $\quad 10, \quad 110-123$. http://www.udgvirtual.udg.mx/apertura/index.php/apertura/article/view/13 $\underline{74}$

Chiecher, A. (2018). Competencias digitales de jóvenes que inician sus trayectorias universitarias ¿Desafios para la docencia en la era digital? https://virtualeduca.red/ISO/2018/data/ponencias/Ponencias2018/Foro\%2 OEducadores\%20para\%20la\%20Era\%20Digital/VEBR18.0096.pdf

Durán, M., Gutiérrez, I., \& Prendes, M. (201). Análisis conceptual de modelos de competencia digital del profesorado universitario. RELATEC: Revista Latinoamericana de Tecnología Educativa, 15(1), 97-114. Doi: https://doi.org/10.17398/1695-288X.15.1.97

Esteve, F; Gisbert, C. y Lázaro, C. (2016). LA COMPETENCIA DIGITAL DE LOS FUTUROS DOCENTES: ¿CÓMO SE VEN LOS ACTUALES 
ESTUDIANTES DE EDUCACIÓN? Red de Revistas Científicas de América Latina, el Caribe, España y Portugal. https://www.redalyc.org/pdf/3333/333346580004.pdf

Gálvez, E. \& Milla, R. (2018). Evaluación del desempeño docente: Preparación para el aprendizaje de los estudiantes en el Marco de Buen Desempeño Docente. Propósitos y Representaciones, 6(2). 407-452 doi: http:// dx.doi.org/10.20511/pyr2018.v6n2.236

Gallardo, E., Marqués, L. y Bullen, M. (2016). Hablemos de aprendices digitales en la era digital. Revista Mexicana de Bachillerato a Distancia, 8(15), 148-182. http://bdistancia.ecoesad.org.mx/?articulo=lets-talk-about-digitallearners-in-thedigital-era.

Gil-Quintana, J. y Cano-Alfaro, A. (2020). Inclusión digital: un reto para la organización, planificación y didáctica escolar. Revista Mediterránea de Comunicación/Mediterranean Journal of Communication, 11(1), 51-60. https://www.doi.org/10.14198/MEDCOM2020.11.1.6

Gisbert, M., González, J. y Esteve, F. (2016). Competencia digital y competencia digital docente: una panorámica sobre el estado de la cuestión. RIITE. Revista Interuniversitaria de Investigación en Tecnología Educativa, 0,7483. Doi: http://dx.doi.org/10.6018/riite/2016/257631

Hernández, Ronald M, Orrego Cumpa, Rosalina, \& Quiñones Rodríguez, Sonia. (2018). Nuevas formas de aprender: La formación docente frente al uso de las TIC. Propósitos y Representaciones, 6(2),671-685. https://dx.doi.org/10.20511/pyr2018.v6n2.248

Iordache, C., Mariën, I., \& Baelden, D. (2017). Developing Digital Skills and Competences: A QuickScan Analysis of 13 Digital Literacy Models. Italian Journal of Sociology of Education, 9(1), 6-30. doi: https://doi.org/10.14658/pupj-ijse-2017-1-2 
Lévano-Francia, L., Sanchez, S., Guillén-Aparicio, P., Tello-Cabello, S., HerreraPaico, N., Collantes-Inga, Z. (2019). Competencias digitales y educación. Propósitos y Representaciones, 7(2), 569-588. doi: http://dx.doi.org/10.20511/pyr2019.v7n2.329

Manzi, J., Gonzáles, R., \& Sun, Y. (2012). La Evaluación Docente en Chile. Santiago de Chile.: Centro de Medición MIDE. https://www.mideuc.cl/libroed/pdf/La_Evaluacion_Docente_en_Chile.pdf

Marza, M., \& Cruz, E. (2018). Gaming como Instrumento Educativo para una Educación en competencias Digitales desde los Academic Skills Centres. Revista General de Información y Documentación, 28(2), 489-506. Doi: http://dx.doi.org/10.5209/RGID.60805

McKnight, K.(2016) Enseñar en una era digital: cómo los educadores usan la tecnología para mejorar el aprendizaje de los estudiantes. Revista Journal of Research on Technology in Education, Edición n48. https://www.researchgate.net/publication/303463215_Teaching_in_a_Digit al_Age_How_Educators_Use_Technology_to_Improve_Student_Learning

Melgar, I. (2015). Calidad de la educación. México, DF: El Sol, S.A. de C.V

Ministerio de Educación. (2012). El Marco del Buen Desempeño Docente. Lima: Ministerio de Educación. http://www.minedu.gob.pe/files/5087_201301030900.pdf

Ocaña-Fernández, Y., Valenzuela-Fernández, L., y Garro-Aburto, L. (2019). Inteligencia artificial y sus implicaciones en la educación superior.

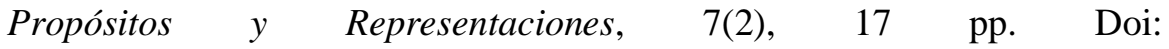
http://dx.doi.org/10.20511/pyr2019.v7n2.274

Olaguibel, G. (2010). Niveles de Desempeño en aula de docentes que participaron y no participaron en el Programa de Capacitación convenio. http://repositorio.usil.edu.pe/bitstream/123456789/1104/1/2010_C\%C3\%A 1rdenas_Nivel\%20de\%20desempe\%C3\%B1o\%20en\%20aula\%20de\%20d 
ocentes\%20que $\% 20$ participaron $\% 20 \mathrm{y} \% 20$ no\%20participaron $\% 20 \mathrm{en} \% 20 \mathrm{el}$ $\% 20$ programa\%20de\%20capacitaci\%C3\%B3n\%20Convenio\%20UNE-

Callao.pdf

Pol, P. (2015) E-Learning un modelo pedagógico. Segunda edición. Editorial INA. San Jose, Costa Rica. https://issuu.com/inavirtual/docs/libro_completo [ Links ]

Rivas, A. (2015). América Latina después de PISA: lecciones aprendidas de la educación en siete países 200-2015. Buenos Aires: Fundación CIPPEC. http://mapeal.cippec.org/wpcontent/uploads/2015/05/Rivas_A_2015_America_Latina_despues_de_PIS $\underline{\text { A.pdf }}$

UNESCO. (2016). Tecnologías digitales al servicio de la calidad educativa. Una propuesta de cambio centrada en el aprendizaje para todos. http://unesdoc.unesco.org/images/0024/002451/245115S.pdf

Vaillant, D. (2016). El fortalecimiento del desarrollo profesional docente: una mirada desde Latinoamérica. Journal of Supranational Policies of $\begin{array}{lllllll}\text { Education, } & n^{o} & 5, & \text { pp. } & 5 & - & 21 .\end{array}$ https://dialnet.unirioja.es/servlet/articulo?codigo $=5691055$ 